\title{
Characteristics of Leg and Ankle in Taxi Drivers
}

\author{
Sun-young Kang ${ }^{1}$, MSc, PT, Sung-dae Choung ${ }^{1}$, BHSc, PT, Hye-seon Jeon ${ }^{2,3}$, PhD, PT \\ ${ }^{1}$ Dept. of Physical Therapy, The Graduate School, Yonsei University \\ ${ }^{2}$ Dept. of Physical Therapy, College of Health Science, Yonsei University \\ ${ }^{3}$ Dept. of Ergonomic Therapy, The Graduate School of Health and Environment, Yonsei University
}

\begin{abstract}
For professional drivers, there is a possibility to have musculoskeletal disorders on ankle joint due to repetitive pedaling operation. Therefore, this study have focused to examine ankle active range of motion (AROM), dorsiflexor strength, and pressure pain threshold (PPT) of tibialis anterior muscle (TA) in taxi drivers compared to a age-matched control group. Thirty male taxi drivers with at least 10 years of driving experience and thirty male sedentary workers were evaluated for ankle AROM, dorsiflexor strength, and PPT of TA. Multiple independent t-tests were used to identify significant differences between two groups. For the results, taxi drivers had significantly less AROM in dorsiflexion and greater AROM in external tibial rotation compared to the control group. Also, dorsiflexor strength and PPT of TA in taxi drivers was significantly lower than in the control group. This study indicates that the repetitive ankle movements associated with driving have an effect on ankle AROM, dorsiflexor strength, and PPT of TA and may lead to work-related musculoskeletal disorders on ankle. Professional drivers may need to be educated to prevent a potential musculoskeletal disorders associated with repetitive movement.
\end{abstract}

Key Words: Ankle; Musculoskeletal disorders; Pressure pain threshold; Range of motion; Strength; Taxi driver.

\section{Introduction}

Professional drivers are defined as workers whose main task is to operate a motor vehicle, such as chauffeurs and bus, truck, tram, trolley, taxi, and ambulance drivers (Bylund et al, 1997). The health of professional drivers is important, as it is a factor in assuring the safety of passengers using the service as well as of the drivers themselves (Winkleby et al, 1988). Based on previous studies, professional drivers are at high risk for sciatica (Heliövaara, 1987), intervertebral disc displacement (Bovenzi and Zadini, 1992; Kelsey and Hardy, 1975), lumbar spine degeneration (Luoma et al, 1998), and non-specific low-back pain (Anderson, 1992; Bovenzi and Zadini, 1992). According to a review of 22 epidemiological studies, musculoskeletal disorders (MSDs) are a pri- mary health issue in drivers (Winkleby et al, 1988). A study of 249 bus drivers showed that $60 \%$ of the participants had symptoms of MSDs, with low back pain as the most common complaint (57\%), followed by leg pain (24\%) and neck pain (21\%) (Costa et al, 2001).

The main factors in MSDs are work of a repetitive, forceful movements and mal-alignment posture (Armstrong et al, 1993). Previous studies have focused on the interior of a car which is related to driving posture, and thus cause MSDs. The interior of a car can be uncomfortable because drivers have to twist their bodies on fixed pelvis to communicate with their passengers, and can be a safety hazard due to tight quarters which make it uneasy for a driver's body to collide with the instruments. Because of this, previous studies have attempted to

Corresponding author: Sung-dae Choung dae282282@hanmail.net 
optimize the position of the pedal, the angle of the seat, and the distance from the center of the wheel to the panel, in order to make driving posture safer and more comfortable (Brook et al, 2009).

In recent times, an automatic transmission has become more popular than a manual transmission due to the simple and convenient operation of an automatic, such as the fact that it only requires one foot to control the accelerator and brake pedals. To increase or decrease speed, drivers control the accelerator or brake pedal, respectively, with repetitive dorsiflexion and plantar flexion movement in the ankle. In stop-and-go traffic, drivers alternate between the accelerator and brake pedal by pivoting the ankle on the heel. These repetitive movements may be associated with a high incidence of MSDs on leg, but there is no study on leg and ankle of professional drivers. Therefore, the purpose of this study is to examine ankle active range of motion (AROM), dorsiflexor strength, and pressure pain threshold (PPT) of tibialis anterior muscle (TA) in taxi drivers compared to a control group. We hypothesized that taxi drivers would have a significant difference ankle AROM, dorsiflexor strength, and PPT of TA compared to the control group.

\section{Methods}

\section{Subjects}

This was a cross-sectional control study with 30 middle aged male taxi drivers and 30 age-matched male sedentary-workers with right leg dominant (Table 1). All taxi drivers in this study met the fol- lowing inclusion criteria: at least 10 years of driving experience with an automatic transmission, and use of only the right foot in pedal operation. The control group consisted of thirty male sedentary-workers who drive less than $30 \mathrm{~km}$ per day. Their job was to assemble car components in a seated posture that does not involve repeated movement of the ankle. The exclusion criteria were the same for both groups: 1) neurological disorders, 2) diabetes, and 3) disorders of the hip, knee, or ankle in the previous six months. Ethical approval was obtained from the Yonsei University Wonju Institutional Review Board and all subjects provided informed written consent.

\section{Measured variables and procedure}

This study measured AROM, PPT of TA, dorsiflexor strength in the right ankle, which is the ankle used to press the accelerator and brake pedals. AROM consisted of dorsiflexion, plantar flexion, eversion, and inversion. All variables were measured by independent examiner having the relevant skills and 5-years experience to undertake a competent examination. In addition, analyze of all variables was performed by independent primary researcher who blinded to the involved groups.

\section{Ankle active range of motion}

AROM of dorsiflexion, plantar flexion, eversion, and inversion angles were recorded by a digital camera and video motion analysis software SIMI $^{\circ}$ Twinner Pro (SIMI Reality Motion Systems GmbH, Unterschleissheim, Germany) was used to analyze the kinematic data. Participants were seated on the edge of a plinth with the lower leg over the bed un-

Table 1. General characteristics of subjects

\begin{tabular}{cccc}
\hline \hline & Taxi drivers $\left(\mathrm{n}_{1}=30\right)$ & Control $\left(\mathrm{n}_{2}=30\right)$ & $\mathrm{p}$ \\
\hline Age $($ year $)$ & $51.4 \pm 4.1^{\mathrm{a}}$ & $49.3 \pm 6.0$ & .150 \\
Weight $(\mathrm{kg})$ & $69.3 \pm 5.8$ & $71.0 \pm 5.5$ & .285 \\
Height $(\mathrm{cm})$ & $170.4 \pm 3.5$ & $171.7 \pm 3.2$ & .142 \\
Body mass index $\left(\mathrm{kg} / \mathrm{m}^{2}\right)$ & $24.0 \pm 2.7$ & $24.0 \pm 2.0$ & .852 \\
\hline
\end{tabular}

${ }^{a}$ mean \pm standard deviation. 
supported, and the ankle in a comfortable relaxed position. Zero starting position was defined as the position in which the heel was aligned with the midline of the tibia and the ankle and knee joint were in a $90^{\circ}$ neutral position. The participants then actively moved their ankle to the end of range of motion, with guidance from the observer. Participants were asked to hold their ankle at the end of range while the image was captured on a digital camera.

Dorsiflexion and plantar flexion angles were measured by placing a camera one meter from the subject's right side with the camera perpendicular to the ground and parallel to the frontal plane of the subject, and the lens approximately level with the subject's lateral malleolus. Markers were placed by the same investigator on the distal third of the fibular, lateral malleolus, and lateral aspect of fifth metatarsal head (Figure 1A). Dorsiflexion and plantar flexion angles were defined as the angle created by the line from the fibular to lateral malleolus and the line from the lateral malleolus to the fifth metatarsal head (Kaufman et al, 1999). Eversion and inversion angles were measured by placing the camera level with the subject's right knee, with the camera perpendicular to the ground and the lens facing the subject's right foot. Markers were placed at the midpoint between the malleoli on the anterior aspect of the ankle, the midline on the anterior aspect of the lower leg using
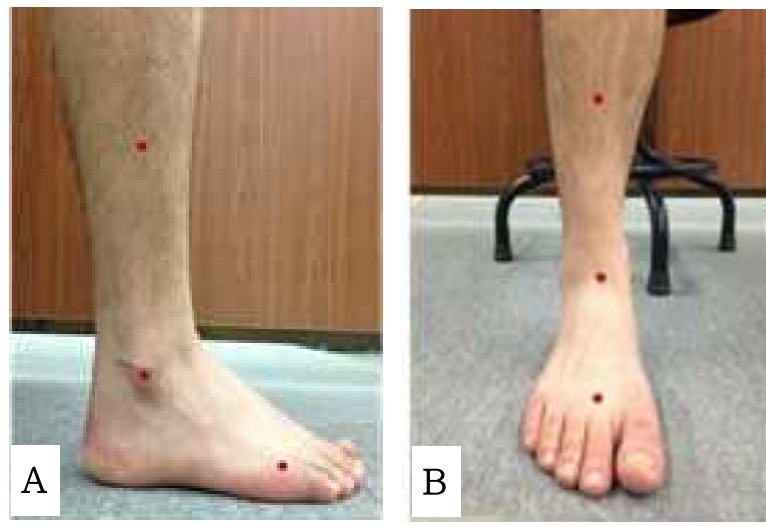

Figure 1. The markers attachment for active range of motion (A: dorsiflexion and plantar flexion, $\mathrm{B}$ : eversion and inversion). the crest of the tibia as a reference point, and the longitudinal midline on the dorsal surface of the second metatarsal (Figure 1B). Eversion and inversion angles were defined as the angle created by the line from the midpoint between the malleoli to the midline on the tibia anterior aspect and the line from the midpoint between the malleoli to the second metatarsal (Spink et al, 2010). High intra-observer reliability has previously been reported for this measuring method (ICC=.82 .96) (Menadue et al, 2006).

External and internal tibial rotation angles were measured using the footprint method. This method is commonly used in clinical settings to estimate underlying tibial rotation and it is the most repeatable method (coefficient of repeatability=5.4) (Hazlewood et $\mathrm{al}, 2007)$. A piece of pre-lined paper was placed under the foot with the lines on the paper parallel to the knee axis (Figure 2). The examiner passively demonstrated the movement required before the test and then asked the participants to actively move their ankle to the end of range of motion. During the movement, the thigh was passively fixed with examiner's hands to prevent any compensate hip movement. Two marks were made vertically below the centers of the malleoli using a small set square. A line was drawn between these two marks, and the external and internal tibial rotation angle was defined as the angle created by the drawn line and the line
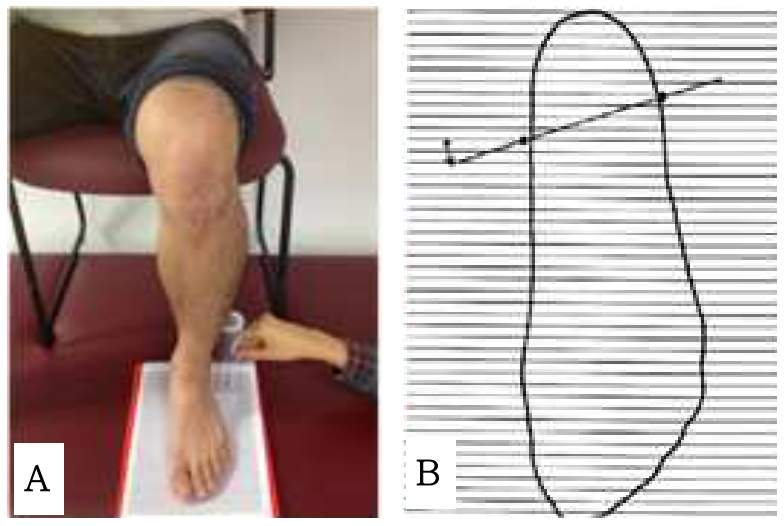

Figure 2. The measurement of external and internal tibial rotation angle (A: the footprint method, B: the footprint drawn on the lined paper). 

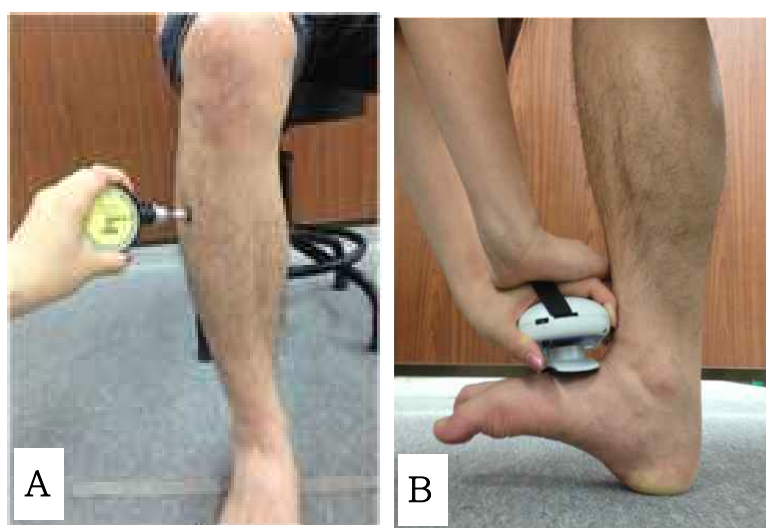

Figure 3. The experimental setting (A: pressure pain threshold measurement of the tibialis anterior muscle, B: strength measurement of the ankle dorsiflexor).

on the paper (Hazlewood et al, 2007).

\section{Pressure pain threshold of tibialis anterior muscle}

A handheld pressure algometer, the FPK 60 (Wagner Instruments Inc., Greenwich, USA), was used to identify the pressure and/or force that elicited pain from the TA. This algometer has demonstrated high reliability and validity (Kinser et al, 2009). The pressure algometer has a force gauge with a $1 \mathrm{~cm}^{2}$ rubber tip and holds at a maximum reading of $25 \mathrm{~kg}$. Subjects were examined in a $\mathrm{re}^{-}$ laxed seated position and standardized instruction was given prior to measurement. Subjects were instructed to "report as soon as the sensation of pressure changes to pain by saying 'stop,' and I will stop." The rubber tip of the algometer was positioned perpendicular to the proximal third of the TA muscle belly (Figure 3A) with the gauge turned away from the subject and the examiner (Collins et al, 2004).

\section{Dorsiflexor strength}

A hand-held dynamometer (Lafayette Instrument Company, North Lafayette, USA) was used to measure dorsiflexor strength. The test was performed with participants in a seated position, holding the dorsiflexion position with their heel on the ground.
The examiner passively demonstrated the movement required and then asked the participants to perform the movement in order to familiarize them with the movement before the test. For the test, the hand-held dynamometer was positioned against the dorsal surface of the foot just proximal to the metatarsal heads (Figure 3B), and the participant exerted maximal force against it (Spink et al, 2010). Three consecutive contractions were recorded and the average was used for analysis (Wang et al, 2002). Details of the test protocol have been reported in a previous study, which reported excellent test-retest for both intra-rater reliability (ICC $=.78 \sim .94)$ and in ${ }^{-}$ terrater reliability (ICC=.77 .88) (Spink et al, 2010).

\section{Statistical analysis}

All statistical analyses were conducted using SPSS ver. 18.0 (SPSS Inc., Chicago, IL, USA). Multiple independent $t$-tests were used to identify significant differences in ankle AROM, dorsiflexor strength, and PPT of TA between taxi drivers and control group. A p-value of less than .05 was considered statistically significant.

\section{Results}

Table 2 shows the ankle AROM in both groups. There were no significant differences in plantar flexion, eversion, inversion, and internal tibial rotation between groups. However, taxi drivers had significantly less AROM in dorsiflexion and significantly greater AROM in external tibial rotation compared to the control group. Table 3 shows dorsiflexor strength and PPT of the TA. Ankle dorsiflexor strength and pain threshold of the TA in taxi drivers were significantly less than in the control group.

\section{Discussion}

Work-related MSDs of the ankle and foot have 
Table 2. Ankle active range of motion

\begin{tabular}{cccc}
\hline \hline & Taxi drivers $\left(\mathrm{n}_{1}=30\right)$ & Control group $\left(\mathrm{n}_{2}=30\right)$ & $\mathrm{p}$ \\
\hline Dorsiflexion $\left(^{\circ}\right)$ & $20.17 \pm 3.60^{\mathrm{a}}$ & $25.51 \pm 3.02$ & $<.001^{*}$ \\
Plantar flexion $\left(^{\circ}\right)$ & $49.97 \pm 8.64$ & $48.54 \pm 4.73$ & .454 \\
Eversion $\left(^{\circ}\right)$ & $21.81 \pm 5.70$ & $19.25 \pm 7.92$ & .180 \\
Inversion $\left(^{\circ}\right)$ & $30.95 \pm 6.09$ & $33.52 \pm 7.38$ & .174 \\
External tibial rotation $\left(^{\circ}\right)$ & $30.93 \pm 6.00$ & $22.66 \pm 6.05$ & $<.001^{*}$ \\
Internal tibial rotation $\left(^{\circ}\right)$ & $14.55 \pm 3.02$ & $14.18 \pm 4.11$ & .708 \\
\hline
\end{tabular}

${ }^{a}$ mean \pm standard deviation, ${ }^{*} \mathrm{p}<.05$.

Table 3. Dorsiflexor strength and pressure pain threshold of the tibialis anterior muscle

\begin{tabular}{cccc}
\hline \hline & Taxi drivers $\left(\mathrm{n}_{1}=30\right)$ & Control group $\left(\mathrm{n}_{2}=30\right)$ & $\mathrm{p}$ \\
\hline Dorsiflexor strength $(\mathrm{kg})$ & $27.34 \pm 4.41^{\mathrm{a}}$ & $33.38 \pm 3.18$ & $<.001$ \\
PPT $^{\mathrm{b}}$ of $\mathrm{TA}^{\mathrm{c}}(\mathrm{kg})$ & $17.56 \pm 4.01$ & $21.72 \pm 3.25$ & $<.001$ \\
\hline
\end{tabular}

${ }^{\mathrm{a}}$ mean \pm standard deviation, ${ }^{\mathrm{b}}$ pressure pain threshold, ${ }^{\mathrm{c}}$ tibialis anterior muscle, ${ }^{*} \mathrm{p}<.05$.

been studied extensively in professional drivers; however, it remains unclear exactly how repetitive braking and accelerating affect the ankles of professional drivers. Therefore, this study aimed to $\mathrm{ex}^{-}$ amine ankle AROM, dorsiflexor strength, and PPT of TA in a group of taxi drivers compared to a control group.

This study demonstrated that only ankle dorsiflexion and external tibial rotation AROM showed significant group different taxi drivers had a smaller angle of ankle dorsiflexion than the control. A previous study examining drivers' perceived comfort in the seated position showed that drivers adjust their seat to maintain $90 \sim 110^{\circ}$ between the calf and foot (Park et al, 2000). This indicates that the repetitive movement of pressing the brake primarily occurs by moving the foot from a neutral position to plantar flexion. Another study showed that most drivers operate the accelerator in plantar flexion, around the midpoint of the maximum voluntary contraction range (Brook et al, 2009). These two previous studies suggested that taxi drivers may have shortened posterior calf muscles due to repetitive plantar flexion movement, which causes limited dorsiflexion $\mathrm{AROM}$ in taxi drivers compared to the control group.

External tibial rotation in taxi drivers was sig- nificantly greater than in the control group. When driving in stop-and-go traffic, drivers alternately step on accelerator and brake pedal by pivoting the ankle on the heel. We may think, from the result in this study, that drivers mainly use external tibial rotation to pivot while switching from the brake to the accelerator pedal, and drivers may position their heel point near the brake pedal. During external tibial rotation, when the tibial tuberosity moves laterally, the patellar tendon pulls laterally on the inferior patella, thus rotating the superior aspect of the patella medially about the center of the patella (Lee et al, 2003). This repetitive external tibial rotation may increase stress on the medial side of the knee and could cause patellofemoral instability and compression syndrome (Fox, 1975; Larson et al, 1978). In addition, biceps femoris and tensor fascia latae, as another factors to cause external tibial rotation, may increase of stiffness, in turn could lead to tibiofemoral rotation syndrome (Kwak et al, 2000; Sahrmann, 2002).

This study focused on the TA muscle, which is located in the anterior part of the tibia and is responsible for dorsiflexion and plantar flexion of the ankle. The TA concentrically contracts when the foot is in dorsiflexion and eccentrically contracts when the foot is in plantar flexion (Netter, 1987). Taxi 
drivers contract the TA to control the brake and $\mathrm{ac}^{-}$ celerator pedal and to hold the ankle in dorsiflexion when waiting for a traffic signal.

In this study, PPT of TA and dorsiflexor strength were lower in taxi drivers than in the control group. Muscles become weak when they maintain a lengthened position, particularly over prolonged periods of time. Stretch weakness is a condition in which a muscle is elongated beyond physiological neutral, but not beyond the normal range of motion (Page et al, 2010). Prolonged muscle elongation causes muscle spindle inhibition and the creation of additional sarcomeres, which eventually shift the length tension curve rightward (Sahrmann, 2002). In addition, muscles strained from overuse are usually painful when palpated or when contracting (Page, 2010). In this study, lower PPT of TA and dorsiflexor strength of taxi drivers suggests that the repetitive movements associated with pressing the brake and accelerator pedals leads to stretch weakness and microtrauma in the TA.

This study has several limitations. Drivers in this study were recruited from only one province. In addition, only taxi drivers who had used an automatic transmission for at least 10 years were included in the study. However, many drivers still use a manual transmission, and they may have different characteristics due to using both feet for pedal operation. Therefore, the study sample may not be representative of the overall driver population and further $\mathrm{re}^{-}$ search with a larger, more diverse study sample maybe necessary. In addition, this study did not $\mathrm{ex}^{-}$ amine kinematic change such as joint moment and force, and physiological change such as electromyography amplitude and median frequency while pedaling movement. In addition, further experimental study including these factors would be worth undertaking to find out the effects of repetitive pedaling movement on the ankle joint and surrounding muscle.

Despite these limitations, the results of this study have potentially important implications for taxi drivers. Occupations involving repetitive movements are associated with a high incidence of MSDs, which include a wide range of inflammatory and degenerative conditions. Work-related MSDs are known to cause symptoms such as pain and numbness, and can lead to reduced worker productivity, lost time from work, and temporary or permanent disability (Lei et al, 2005). This study indicates that the repetitive ankle movements associated with operating the brake and accelerator pedal may have an effect on ankle AROM, dorsiflexor strength, and PPT of TA and could lead to work-related MSDs.

\section{Conclusion}

Previous research involving professional drivers has focused on seating comfort, with less focus on how the foot and ankle are affected by operation of the pedals. This study examined how the repetitive motion of operating the brake and accelerator pedals impacts the ankle. Taxi drivers showed decreased ankle dorsiflexion AROM, increased tibial external rotation AROM, and decreased dorsiflexor strength and PPT of TA compared to the control group. This indicates that the repetitive motion associated with operating the brake and accelerator pedals alters ankle AROM, dorsiflexor strength, and PPT of TA. Preventive measures such as allowing adequate rest and exercise for drivers will ultimately ensure the safety of passengers using this service as well as the drivers themselves.

\section{References}

Anderson R. The back pain of bus drivers. Prevalence in an urban area of California. Spine (Phila Pa 1976). 1992;17(12):1481-1488.

Armstrong TJ, Buckle P, Fine LJ, et al. A conceptual model for work-related neck and upper-limb musculoskeletal disorders. Scand J 
Work Environ Health. 1993;19(2):73-84.

Bovenzi M, Zadini A. Self-reported low back symptoms in urban bus drivers exposed to whole-body vibration. Spine (Phila $\mathrm{Pa}$ 1976). 1992;17(9):1048-1059.

Brook S, Freeman R, Rosala G, et al. Ergonomic data measuring system for driver-pedals interaction. SAE Int J Passeng Cars - Mech Syst. 2009;2(1):1071-1078.

Bylund PO, Björnstig U, Larsson TJ. Occupational road trauma and permanent medical impairment. Saf Sci. 1997;26(3):187-200.

Collins N, Teys P, Vicenzino B. The initial effects of a mulligan's mobilization with movement technique on dorsiflexion and pain in subacute ankle sprains. Man Ther. 2004;9(2):77-82.

Costa G, Sartori S, Facco P, et al. Health conditions of bus drivers in a 6 year follow up study. J Hum Ergol (Tokyo). 2001;30(1-2):405-410.

Fox TA. Dysplasia of the quadriceps mechanism: Hypoplasia of the vastus medialis muscle as $\mathrm{re}^{-}$ lated to the hypermobile patella syndrome. Surg Clin North Am. 1975;55(1):199-226.

Hazlewood ME, Simmons AN, Johnson WT, et al. The footprint method to assess transmalleolar axis. Gait Posture. 2007;25(4):597-603.

Heliövaara M. Occupation and risk of herniated lumbar intervertebral disc or sciatica leading to hospitalization. J Chronic Dis. 1987;40(3):259-264.

Kaufman KR, Brodine SK, Shaffer RA, et al. The effect of foot structure and range of motion on musculoskeletal overuse injuries. Am J Sports Med. 1999;27(5):585-593.

Kelsey JL, Hardy RJ. Driving of motor vehicles as a risk factor for acute herniated lumbar intervertebral disc. Am J Epidemiol. 1975;102(1):63-73.

Kinser AM, Sands WA, Stone MH. Reliability and validity of a pressure algometer. J Strength Cond Res. 2009;23(1):312-314.

Kwak SD, Ahmad CS, Gardner TR, et al. Hamstrings and iliotibial band forces affect knee kinematics and contact pattern. J Orthop Res.
2000;18(1):101-108.

Larson RL, Cabaud HE, Slocum DB, et al. The patellar compression syndrome: Surgical treatment by lateral retinacular release. Clin Orthop Relat Res. 1978;134:158-167.

Lee TQ, Morris G, Csintalan RP. The influence of tibial and femoral rotation on patellofemoral contact area and pressure. J Orthop Sports Phys Ther. 2003;33(11):686-693.

Lei L, Dempsey PG, Xu JG, et al. Risk factors for the prevalence of musculoskeletal disorders among chinese foundry workers. Int $\mathrm{J}$ Ind Ergon. 2005;35(3):197-204.

Luoma K, Riihimäki H, Raininko R, et al. Lumbar disc degeneration in relation to occupation. Scand J Work Environ Health. 1998;24(5):358-366.

Netter FH. The Ciba Collection of Medical Illustrations, Vol. 8, Musculoskeletal System, Part I. Anatomy, physiology and metabolic disorders. 1987:98-101.

Menadue C, Raymond J, Kilbreath SL, et al. Reliability of two goniometric methods of measuring active inversion and eversion range of motion at the ankle. BMC Musculoskelet Disord. 2006;7:60.

Page P, Frank C, Lardner R. Assessment and Treatment of Muscle Imbalance: The Janda approach. 1st ed. Champaign, IL, Human Kinetics, 2010:48-52.

Park SJ, Kim CB, Kim CJ, et al. Comfortable driving postures for Koreans. Int $\mathrm{J}$ Ind Ergon. 2000;26(4):489-497.

Sahrmann S. Diagnosis and Treatment of Movement Impairment Syndromes. 1st ed. St. Louis, Mosby, 2002:36-41.

Spink MJ, Fotoohabadi MR, Menz HB. Foot and ankle strength assessment using hand-held dynamometry: Reliability and age-related differences. Gerontology. 2010;56(6):525-532.

Wang CY, Olson SL, Protas EJ. Test-retest strength reliability: Hand-held dynamometry in community-dwelling elderly fallers. Arch Phys Med 
한국전문물리치료학회지 2014년 21권 1호 55-62

Phys Ther Kor 2014;21(1):55-62

Rehabil. 2002;83(6):811-815.

Winkleby MA, Ragland DR, Fisher JM, et al. Excess risk of sickness and disease in bus drivers: A review and synthesis of epidemiological studies. Int J Epidemiol. 1988;17(2):255-262.
This article was received November 25, 2013, was reviewed November 25, 2013, and was accepted February 3, 2014. 